\title{
Infrared and Ultraviolet Dimensional Meromorphy of Feynman Amplitudes
}

\author{
C. de Calan and A. P. C. Malbouisson* \\ Centre de Physique Théorique de l'Ecole Polytechnique, Plateau de Palaiseau, F-91128 Palaiseau \\ Cedex, France
}

\begin{abstract}
By the concurrent use of dimensional and analytic regularizations with the complete Mellin (CM) representation, we find in a direct way the ultraviolet and infrared poles in space-time dimension, for any Feynman amplitude with an arbitrary subset of vanishing masses.
\end{abstract}

\section{Introduction}

A dimensionally regularized Feynman amplitude [1] is the analytic continuation, in the space-time dimension $D$, of the function $A_{G}(D)$ defined by the Feynman integral corresponding to a given graph $G$. When there is no vanishing internal mass, it is well known that the integral exists for a sufficiently low value of $\operatorname{Re} D$, and defines a meromorphic function of $D$ : the singularities of $A_{G}(D)$ are poles, located at real rational values of $D$. We denote by $D_{\mathrm{UV}}$ the first pole, that is the lowest value of $D$ for which the Feynman integral presents ultraviolet (UV) divergences.

Now if there are vanishing masses, the Feynman integral may present infrared (IR) divergences for $\operatorname{Re} D \leqq D_{\mathrm{IR}}$. When all masses vanish, it has been shown that $A_{G}(D)$ remains meromorphic, with new "infrared" poles [2]. But in the literature there seems to be no such result for the Feynman integrals with only a partial subset of vanishing masses: here we prove the meromorphy of $A_{G}(D)$ in this more general situation.

In Sect. II we look at the case $D_{\mathrm{IR}}<D_{\mathrm{UV}}$. Then $A_{G}(D)$ is defined by the Feynman integral for $D_{\mathrm{IR}}<\operatorname{Re} D<D_{\mathrm{UV}}$. And we use the CM representation [3] to prove that the singularities for $\operatorname{Re} D \leqq D_{\mathrm{IR}}$ are still poles, located at real rational values of $D$.

In the case $D_{\mathrm{IR}} \geqq D_{\mathrm{UV}}$, the formal Feynman integral exists nowhere. In Sect. III, we use the analytic regularization [4] to define $A_{G}(D)$, and we extend the results of Sect. II to this case.

* Permanent address: Centro Brasileiro de Pesquisas Fisicas, new official address: Rua Dr. Xavier Sigaud, 150, URCA, 22.290 Rio de Janeiro RJ, Brasil 
We consider the present paper as a first step towards a better description of the infrared divergences. Our following goal would be an explicit CM representation of the dimensionally renormalized amplitudes, with an arbitrary number of vanishing masses. Then we could try to isolate the whole set of infrared poles in dimension, and to organize their residues, for the physically relevant models like Q.E.D. or Q.C.D.

\section{The Convergent Case}

For the sake of simplicity, we restrict ourselves to the study of scalar amplitudes. The extension to spinor amplitudes or derivative couplings is merely technical, and has been sketched in [5].

If $D_{\mathrm{IR}}<D_{\mathrm{UV}}$, we define $A_{G}(D)$, for $D_{\mathrm{IR}}<\operatorname{Re} D<D_{\mathrm{UV}}$, by its CM representation [3]. Let the index $i$ label the internal lines of a given Feynman graph $G, E$ the set of the massive lines $\left(m_{i} \neq 0 \forall i \in E\right), F$ the set of the massless lines $\left(m_{i}=0 \forall i \in F\right)$. If the index $j$ labels the "one-trees" of the graph and the index $k$ the "two-trees" (with the cut-invariants $s_{k}$ ), we have:

$$
A_{G}(D)=\int_{\Delta \cap P(D)} \frac{\prod_{j} \Gamma\left(-x_{j}\right)}{\Gamma\left(-\sum_{j} x_{j}\right)} \cdot \prod_{k} s_{k}^{y_{k}} \Gamma\left(-y_{k}\right) \cdot \prod_{i \in E}\left(m_{i}^{2}\right)^{-\varphi_{\imath}} \Gamma\left(\varphi_{i}\right) .
$$

The integration variables are the imaginary parts of the variables $x_{j}, y_{k}$ linked by the conditions:

$$
\begin{gathered}
\Delta=\left\{x, y \mid \varphi_{i}=0 \forall i \in F ; \operatorname{Re} x_{j}<0 ; \operatorname{Re} y_{k}<0 ; \operatorname{Re} \varphi_{i}>0 \forall i \in E\right\}, \\
P(D)=\left\{x, y \mid \sum_{j} x_{j}+\sum_{k} y_{k}+\frac{D}{2}=0\right\},
\end{gathered}
$$

where

$$
\varphi_{i}=\sum_{j} u_{i j} x_{j}+\sum_{k} u_{i k} y_{k}+1,
$$

and $u_{i j}\left(u_{i k}\right)=0$ or 1 according to whether the line $i$ belongs or does not belong to the one-tree $j$ (two-tree $k$ ). The convergence condition $D_{\mathrm{IR}}<D_{\mathrm{UV}}$ is equivalent to $\Delta \neq \emptyset$, and we have:

$$
\begin{gathered}
D_{\mathrm{IR}}=\operatorname{Inf}_{\Delta}\left(-2 \operatorname{Re} \sum_{j} x_{j}-2 \operatorname{Re} \sum_{k} y_{k}\right), \\
D_{\mathrm{UV}}=\operatorname{Sup}_{\Delta}\left(-2 \operatorname{Re} \sum_{j} x_{j}-2 \operatorname{Re} \sum_{k} y_{k}\right) .
\end{gathered}
$$

In the same way that any asymptotic expansion can be determined [3], the dimensional singularities are found by translating the integration path in (1). For the ultraviolet poles, this method is explained in [5]. But the same procedure applies as well to the infrared singularities. Let us denote by $\varphi_{v}$ the linear forms $-x_{j},-y_{k}, \varphi_{i}(i \in E)$. From (2), (3), and (5) there exist positive real numbers $c_{v}$ such 
that

$$
\sum_{v} c_{v} \varphi_{v} \equiv D-D_{\mathrm{IR}}
$$

and we have

$$
\prod_{v} \frac{1}{\varphi_{v}}=\frac{1}{D-D_{\mathrm{IR}}} \sum_{v} \frac{c_{v}}{\prod_{v^{\prime} \neq v} \varphi_{v^{\prime}}} .
$$

If there exist again convex generations of $D-D_{\mathrm{IR}}$ by some subsets $\left\{\varphi_{v^{\prime}}, v^{\prime} \neq v\right\}$, the procedure is iterated until we find:

$$
\prod_{v} \frac{1}{\varphi_{v}}=\sum_{H} \frac{c_{H}}{\left(D-D_{\mathrm{IR}}\right)^{q_{H}}} \prod_{v \in H} \frac{1}{\varphi_{v}},
$$

where the subsets $\left\{\varphi_{v}, v \in H\right\}$ no longer generate $D-D_{\mathrm{IR}}$. The family of sets $H$, with the corresponding powers $q_{H}$ and coefficients $c_{H}$, is thus determined by the convex geometry of the linear forms $\varphi_{v}$.

Replacing $\Gamma\left(\varphi_{v}\right)$ in (1) by $1 / \varphi_{v} \cdot \Gamma\left(\varphi_{v}+1\right)$, we obtain

$$
A_{G}(D)=\sum_{H} \frac{c_{H}}{\left(D-D_{\mathrm{IR}}\right)^{q_{H}}} \int_{\Delta_{H} \cap P(D)} \frac{\prod_{k} s_{k}^{y_{k}} \prod_{i}\left(m_{i}^{2}\right)^{-\varphi_{i}}}{\Gamma\left(-\sum_{j} x_{j}\right)} \prod_{v} \Gamma\left(\varphi_{v}+1-\theta_{v H}\right),
$$

where $\theta_{v H}=1$ for $v \in H, 0$ for $v \notin H$,

$$
\Delta_{H}=\left\{x, y \mid \varphi_{i}=0 \forall i \in F ; \operatorname{Re}\left(\varphi_{v}+1-\theta_{v H}\right)>0\right\} .
$$

Since the $\varphi_{v}, v \in H$, no longer generate $D-D_{\mathrm{IR}}$, we have

$$
\operatorname{lnf}_{\Delta_{H}}\left(-2 \operatorname{Re} \sum_{j} x_{j}-2 \operatorname{Re} \sum_{k} y_{k}\right)=D_{\mathrm{IR}}^{H}<D_{\mathrm{IR}},
$$

and the integrals in (9) are analytic in a larger domain in $D$. The same technique can be used to determine the following poles at $D=D_{\mathrm{IR}}^{H}$, etc... Since the coefficients of the linear forms $\varphi_{v}$ are 0 or 1 , the numbers $c_{v}$, in each identity like (7), are rational, and by construction we find the infrared poles at the rational values

$$
D_{\mathrm{IR}}^{\{n\}}=D_{\mathrm{IR}}-\sum_{v} c_{v} n_{v}
$$

where the $n_{v}$ 's are positive integers.

Similarly we have for the lowest ultraviolet pole at $D=D_{\mathrm{UV}}$ a family of identities like

$$
\sum_{v} d_{v} \varphi_{v} \equiv D_{\mathrm{UV}}-D
$$

where the $d_{v}$ 's are positive rational numbers, and the ultraviolet poles are at the rational values

$$
D_{\mathrm{UV}}^{\{n\}}=D_{\mathrm{UV}}+\sum_{v} d_{v} n_{v}
$$




\section{The Divergent Case}

If $D_{\mathrm{IR}} \geqq D_{\mathrm{UV}}$, the domain $\Delta$ is empty and the formal Feynman integral diverges for every value of $D$. In this case we introduce the analytic regularization of Speer [4]. This amounts to replacing each $\varphi_{i}$ by $\varphi_{i}^{\prime}=\varphi_{i}+t_{i}$ in the CM representation. Except for the purely homogeneous integrals $\int d^{m} k k^{p}$, which disappear after renormalization, it is always possible to find a set of parameters $t_{i}$ such that the new domain

$$
\Delta^{\prime}=\left\{x, y \mid \varphi_{i}^{\prime}=0 \forall i \in F ; \operatorname{Re} x_{j}<0 ; \operatorname{Re} y_{k}<0 ; \operatorname{Re} \varphi_{i}^{\prime}>0 \forall i \in E\right\}
$$

is not empty, that is $D_{\mathrm{IR}}(t)<D_{\mathrm{UV}}(t)$.

Then the preceding method applies. With the same sets of positive coefficients $\left\{c_{v}\right\},\left\{d_{v}\right\}$, and for any $t_{i}$ 's, we obtain the infrared and ultraviolet poles at:

$$
\begin{gathered}
D_{\mathrm{IR}}^{\{n\}}(t)=D_{\mathrm{IR}}(t)-\sum_{v} c_{v} n_{v}, \\
D_{\mathrm{UV}}^{\{n\}}(t)=D_{\mathrm{UV}}(t)+\sum_{v} d_{v} n_{v} .
\end{gathered}
$$

Once these poles are explicitly factorized in $A_{G}(D, t)$, as in (9), we come back to $t_{i}=0$. In this way, even when $D_{\mathrm{IR}}(0)=D_{\mathrm{IR}} \geqq D_{\mathrm{UV}}(0)=D_{\mathrm{UV}}$, the amplitude $A_{G}(D)$ is defined and shown to be meromorphic.

Now of course some infrared poles may coincide with ultraviolet ones. At a given value $D_{1}$ of the dimension $D$, the dimensional renormalization will suppress only the ultraviolet poles. The possible remaining infrared poles express the infrared divergences, which we intend to organize, for some specific models, in later papers.

Acknowledgements. One of us (C. C.) is grateful to Professor Tiomno for his hospitality at C.B.P.F., where this work was performed. We also thank F. David for a useful discussion.

\section{References}

1. Speer, E., Westwater, M.: Generic Feynman amplitudes. Ann. Inst. Henri Poincaré A 14, 1 (1971) Bollini, C.G., Giambiagi, J.J.: Dimensional renormalization: the number of dimensions as a regularizing parameter. Nuov. Cimento 12, 1, 21 (1972); and Lowest order "divergent" graphs in $v$-dimensional space. Phys. Lett. B40, 566 (1972)

't Hooft, G., Veltmann, M. : Regularization and renormalization of gauge fields. Nucl. Phys. B44, 189 (1972)

de Vega, H., Schaposnik, F.: Dimensional renormalization. J. Math. Phys. 15, 1998 (1974)

2. Parisi, G. : Nucl. Phys. B150, 163 (1979)

Bergère, M.C., David, F.: Preprint DPhT 82-16, CEN Saclay (1982)

3. de Calan, C., Malbouisson, A.P.C.: Complete Mellin representation and asymptotic behaviours of Feynman amplitudes. Ann. Inst. Henri Poincaré 32, 91 (1980)

4. Bollini, C.G., Giambiagi, J.J., Gonzáles Domínguez, A.: Analytic regularization and the divergences of quantum field theories. Nuov. Cimento 31, 550 (1964)

Speer, E.: Analytic renormalization. J. Math. Phys. 9, 1404 (1968)

Speer, E.: Generalized Feynman amplitudes. Princeton, NJ : Princeton University Press 1969

5. de Calan, C., David, F., Rivasseau, V.: Renormalization in the complete Mellin representation of Feynman amplitudes. Commun. Math. Phys. 78, 531 (1981)

Communicated by J. Lascoux

Received October 1, 1982; in revised form May 1, 1983 